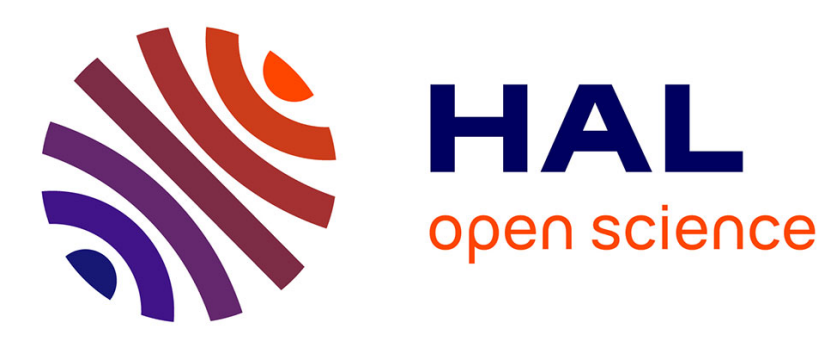

\title{
Assessing the interestingness of temporal rules with Sequential Implication Intensity
}

\author{
Julien Blanchard, Fabrice Guillet, Régis Gras
}

\section{To cite this version:}

Julien Blanchard, Fabrice Guillet, Régis Gras. Assessing the interestingness of temporal rules with Sequential Implication Intensity. Régis Gras, Einoshin Suzuki, Fabrice Guillet, Filippo Spagnolo. Statistical Implicative Analysis, Springer, pp.55-72, 2008, Studies in Computational Intelligence, 10.1007/978-3-540-78983-3_3. hal-00420993

\section{HAL Id: hal-00420993 https://hal.science/hal-00420993}

Submitted on 30 Sep 2009

HAL is a multi-disciplinary open access archive for the deposit and dissemination of scientific research documents, whether they are published or not. The documents may come from teaching and research institutions in France or abroad, or from public or private research centers.
L'archive ouverte pluridisciplinaire HAL, est destinée au dépôt et à la diffusion de documents scientifiques de niveau recherche, publiés ou non, émanant des établissements d'enseignement et de recherche français ou étrangers, des laboratoires publics ou privés. 


\title{
Assessing the interestingness of temporal rules with Sequential Implication Intensity
}

\author{
Julien Blanchard, Fabrice Guillet, and Régis Gras \\ Knowledge \& Decision (KOD) research team \\ LINA - FRE CNRS 2729 \\ Polytechnic School of Nantes University, France \\ julien.blanchard@polytech.univ-nantes.fr
}

\begin{abstract}
In this article, we study the assessment of the interestingness of sequential rules (generally temporal rules). This is a crucial problem in sequence analysis since the frequent pattern mining algorithms are unsupervised and can produce huge amounts of rules. While association rule interestingness has been widely studied in the literature, there are few measures dedicated to sequential rules. Continuing with our work on the adaptation of implication intensity to sequential rules, we propose an original statistical measure for assessing sequential rule interestingness. More precisely, this measure named Sequential Implication Intensity (SII) evaluates the statistical significance of the rules in comparison with a probabilistic model. Numerical simulations show that $S I I$ has unique features for a sequential rule interestingness measure.
\end{abstract}

\section{Introduction}

Frequent pattern discovery in sequences of events ${ }^{1}$ (generally temporal sequences) is a major task in data mining. Research work in this domain consists of two approaches:

- discovery of frequent episodes in a long sequence of events (approach initiated by Mannila, Toivonen, and Verkamo [1] [2]),

- discovery of frequent sequential patterns in a set of sequences of events (approach initiated by Agrawal and Srikant [3] [4]).

The similarity between episodes and sequential patterns is that they are sequential structures, i.e., a structure defined with an order (partial or total). Such a structure can be, for example:

$$
\text { breakfast then lunch then dinner }
$$

The structure is described by its frequency (or support) and generally by constraints on the event position, like a maximal time window "less than 12 hours stand between breakfast and dinner" [4] [5] [6] [7] [8].

\footnotetext{
${ }^{1}$ Here we speak about sequences of qualitative variables. Such sequences are generally not called time series.
} 
The difference between episodes and sequential patterns lies in the measure of their frequency: frequency of episodes is an intra-sequence notion [5] [6] [9] [7] [8] [10], while frequency of sequential patterns is an inter-sequence notion [3] [4] [11] [12] [13] (see [14] for a synthesis on the different ways of assessing frequency). Thus, the frequent episode mining algorithms search for structures which often recur inside a single sequence. On the other hand, the frequent sequential pattern mining algorithms search for structures which recur in numerous sequences (independently of the repetitions in each sequence). These last algorithms are actually an extension to sequential data of the frequent itemset mining algorithms, used among other things to generate association rules [15] [16].

Just as the discovery of frequent itemsets leads to the generation of association rules, the discovery of episodes/sequential patterns is often followed by a sequential rule generation stage which enables predictions to be made within the limits of a time window [4] [5] [6] [11] [12] [9] [7] [8]. Such rules have been used to predict, for example, stock market prices [6] or events in a telecommunication network [5] [8]. A sequential rule can be for instance:

$$
\text { breakfast } \stackrel{6 \mathrm{~h}}{\longrightarrow} \text { lunch }
$$

This rule means "if one observe breakfast then one will certainly observe lunch less than 6 hours later".

In this article, we study the assessment of the interestingness of sequential rules. This is a crucial problem in sequence analysis since the frequent pattern mining algorithms are unsupervised and can produce a huge number of rules. While association rule interestingness has been widely studied in the literature (see [17] for a survey), there are few measures dedicated to sequential rules. In addition to frequency, one mainly finds an index of confidence (or precision) that can be interpreted as an estimation of the conditional probability of the conclusion given the condition [4] [5] [6] [11] [12] [9] [7] [8]. A measure of recall is sometimes used too; it can be interpreted as an estimation of the conditional probability of the condition given the conclusion [9] [8]. In [6] and [7], the authors have proposed an adaptation to sequential rules of the J-measure of Smyth and Goodman, an index coming from mutual information ${ }^{2}$. Finally, an entropic measure is presented in [10] to quantify the information brought by an episode in a sequence, but this approach only deals with episodes and not with prediction rules.

These measures have several limits. First of all, the J-measure is not very intelligible since it gives the same value to a rule $a \stackrel{\omega}{\longrightarrow} b$ and to its opposite $a \stackrel{\omega}{\longrightarrow} \bar{b}$, whereas these two rules make conflicting predictions. Confidence and recall, vary linearly, which makes them rather sensitive to noise. Above all, these measures increase with the size of the time window chosen. This behavior is absolutely counter-intuitive since a rule with a too large time window does not contribute to making good quality predictions. Indeed, the larger the time window, the greater the probability of observing the conclusion which follows

\footnotetext{
${ }^{2}$ The J-measure is the part of the average mutual information relative to the truth of the condition.
} 
the condition in data, and the less significant the rule. Another major problem, which concerns confidence, recall, and J-measure, is that these indexes are all frequency-based: the phenomena studied in data are considered only in a relative way (by means of frequencies) and not in an absolute way (by means of cardinalities). Thus, if a sequence is made longer by repeating it $x$ times one after the other, the indexes do not vary ${ }^{3}$. Statistically, the rules are all the more reliable since they are assessed on long sequences yet. In the end, a good interestingness measure for sequential rules should therefore decrease when the size of the time window is too large, and increase with sequence enlargement. These essential properties have never been highlighted in the literature.

Continuing with our work begun in [18], on the adaptation of implication intensity to sequential rules [19] [20], we propose in this article an original statistical measure for assessing sequential rule interestingness. More precisely, this measure evaluates the statistical significance of the rules in comparison with a probabilistic model. The next section is dedicated to the formalization of the notions of sequential rule, example of a rule, and counter-example of a rule, and to the presentation of the new measure, named Sequential Implication Intensity $(S I I)$. In section 3 , we study $S I I$ in several numerical simulations and compare it to other measures.

\section{Measuring the statistical significance of sequential rules}

\subsection{Context}

Our measure, $S I I$, evaluates sequential rules extracted from one unique sequence. This approach can be easily generalized to several sequences, for example by computing an average or minimal $S I I$ on the set of sequences. Rules are of the form $a \stackrel{\omega}{\longrightarrow} b$, where $a$ and $b$ are episodes (these ones can even be structured by intra-episode time constraints). However, in this article, we restrict our study to sequential rules where the episodes $a$ and $b$ are two single events.

The studied sequence is a continuous sequence of instantaneous events (adaptation to discrete sequences is trivial). It is possible that two different events occur at the same time. This amounts to using the same framework as the one introduced by Mannila, Toivonen, and Verkamo [5]. To extract the appropriate cardinalities from the sequence and compute $S I I$, one only needs to apply their episode mining algorithm named Winepi [1] [5] (or one of its variants). In the following, we stand at the post-processing stage by considering that Winepi has already been applied on the sequence, and we directly work on the episode cardinalities that have been discovered. Here again, our approach could be generalized to other kinds of sequences, for which other episode mining algorithms have been proposed. For example, Höppner has studied sequences with time-interval events that have a non-zero duration and can overlap [7].

\footnotetext{
${ }^{3}$ We consider here that the size of the time window is negligible compared to the size of the sequence, and we leave aside the possible side effects which could make new patterns appear overlapping the end of a sequence and the beginning of the following repeated sequence.
} 


\section{$2.2 \quad$ Notations}

\section{sequence S}

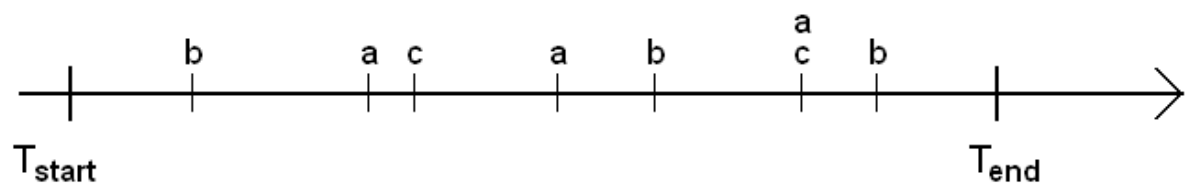

\section{window $\mathbf{F}$}

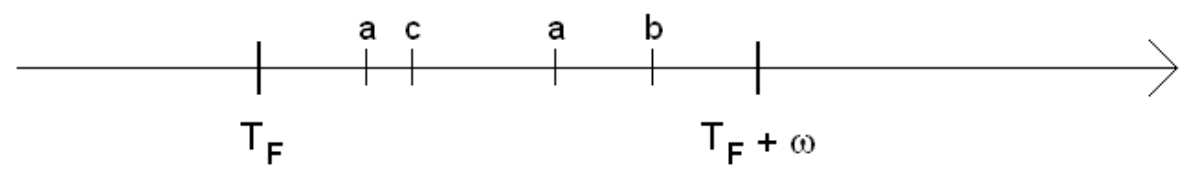

Fig. 1. A sequence $S$ of events from $E=\{a, b, c\}$ and its window $F$ of size $\omega$ beginning at $T_{F}$.

Let $E=\{a, b, c \ldots\}$ be a finite set of event types. An event is a couple $(e, t)$ where $e \in E$ is the type of the event and $t \in \mathbb{R}_{+}$is the time the event occurred. It must be noted that the term event is often used to refer the event type without reducing intelligibility.

An event sequence $S$ observed between the instants $T_{\text {start }}$ and $T_{\text {end }}$ is a finite series of events

$$
S=\left(\left(e_{1}, t_{1}\right),\left(e_{2}, t_{2}\right),\left(e_{3}, t_{3}\right), \ldots\left(e_{n}, t_{n}\right)\right)
$$

such that:

$$
\begin{gathered}
\forall i \in\{1 . . n\},\left(e_{i} \in E \wedge t_{i} \in\left[T_{\text {start }}, T_{\text {end }}\right]\right) \\
\forall i \in\{1 . . n-1\}, t_{i} \leq t_{i+1} \\
\forall(i, j) \in\{1 . . n\}^{2}, t_{i}=t_{j} \Rightarrow e_{i} \neq e_{j}
\end{gathered}
$$

The size of the sequence is $L=T_{\text {end }}-T_{\text {start }}$.

A window on a sequence $S$ is a subsequence of $S$. For instance, a window $F$ of size $\omega \leq L$ beginning at the instant $t_{F} \in\left[T_{\text {start }}, T_{\text {end }}-\omega\right]$ contains all the events $\left(e_{i}, t_{i}\right)$ from $S$ such as $t_{F} \leq t_{i} \leq t_{F}+\omega$.

In the following, we consider a sequence $S$ of events from $E$. 


\subsection{Sequential rules}

We establish a formal framework for sequence analysis by defining the notions of sequential rule, example of a rule, and counter-example of a rule. The examples and counter-examples of a sequential rule have never been defined in the literature about sequences.

\section{sequence $S$}

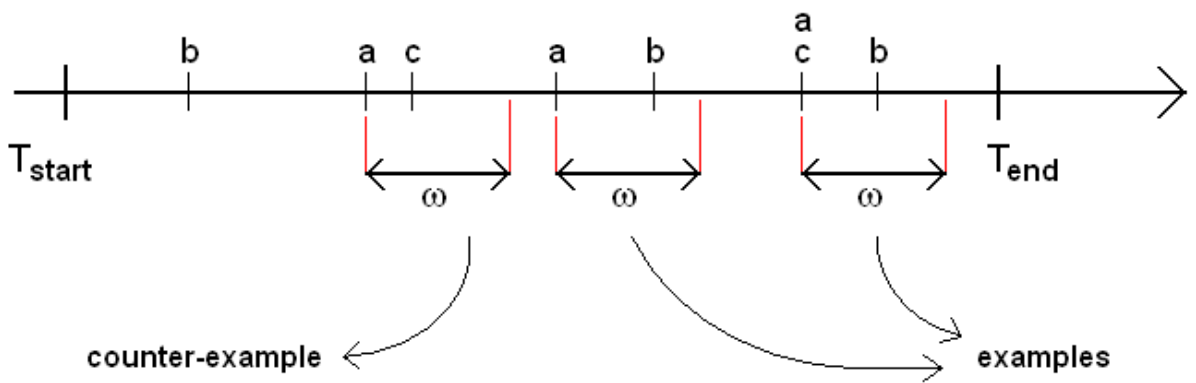

Fig. 2. Among the 3 windows of size $\omega$ beginning on events $a$, one can find 2 examples and 1 counter-example of the rule $a \stackrel{\omega}{\longrightarrow} b$.

Definition 1 A sequential rule is a triple $(a, b, \omega)$ noted $a \stackrel{\omega}{\longrightarrow} b$ where $a$ and $b$ are events of different types and $\omega$ is a strictly positive real number. It means: "if an event $a$ appears in the sequence then an event $b$ certainly appears within the next $\omega$ time units".

Definition 2 The examples of a sequential rule $a \stackrel{\omega}{\longrightarrow} b$ are the events $a$ which are followed by at least one event $b$ within the next $\omega$ time units. Therefore the number of examples of the rule is the cardinality noted $n_{a b}(\omega)$ :

$$
n_{a b}(\omega)=|(a, t) \in S| \exists\left(b, t^{\prime}\right) \in S, 0 \leq t^{\prime}-t \leq \omega \mid
$$

Definition 3 The counter-examples of a sequential rule $a \stackrel{\omega}{\longrightarrow} b$ are the events $a$ which are not followed by any event $b$ during the next $\omega$ time units. Therefore the number of counter-examples of the rule is the cardinality noted $n_{a \bar{b}}(\omega)$ :

$$
n_{a \bar{b}}(\omega)=|(a, t) \in S| \forall\left(b, t^{\prime}\right) \in S,\left(t^{\prime}<t \vee t^{\prime}>t+\omega\right) \mid
$$

Contrary to association rules, $n_{a b}$ and $n_{a \bar{b}}$ are not data constants but depend on the parameter $\omega$. 
The originality of our approach is that it treats condition and conclusion in very different ways: the events $a$ are used as references for searching the events $b$, i.e. only the windows which begin by an event $a$ are taken into account. On the contrary, in the literature about sequences, the algorithms like Winepi move a window forward (with a fixed step) over the whole sequence [5]. This method amounts to considering as examples of the sequential rule any window that has an event $a$ followed by $b$, even if it does not start by en event $a$. In comparison, our approach is algorithmically less complex.

Let us note $n_{a}$ the number of events $a$ in the sequence. We have the usual equality $n_{a}=n_{a b}+n_{a \bar{b}}$. A sequential rule $a \stackrel{\omega}{\longrightarrow} b$ is completely described by the quintuple $\left(n_{a b}(\omega), n_{a}, n_{b}, \omega, L\right)$. The examples of a sequential rule now being defined, we can specify our measure for the frequency of the rules:

Definition 4 The frequency of a sequential rule $a \stackrel{\omega}{\longrightarrow} b$ is the proportion of examples compared to the size of the sequence:

$$
\operatorname{frequency}(a \stackrel{\omega}{\longrightarrow} b)=\frac{n_{a b}(\omega)}{L}
$$

With these notations, the confidence, recall, and J-measure are given by the following formula:

$$
\begin{gathered}
\operatorname{confidence}(a \stackrel{\omega}{\longrightarrow} b)=\frac{n_{a b}(\omega)}{n_{a}} \\
\operatorname{recall}(a \stackrel{\omega}{\longrightarrow} b)=\frac{n_{a b}(\omega)}{n_{b}} \\
J-\text { measure }(a \stackrel{\omega}{\longrightarrow} b)=\frac{n_{a b}(\omega)}{L} \log _{2} \frac{n_{a b}(\omega) L}{n_{a} n_{b}}+\frac{n_{a \bar{b}}(\omega)}{L} \log _{2} \frac{n_{a \bar{b}}(\omega) L}{n_{a}\left(L-n_{b}\right)}
\end{gathered}
$$

\subsection{Random model}

Following the implication intensity for association rules [19], the sequential implication intensity $S I I$ measures the statistical significance of the rules $a \stackrel{\omega}{\longrightarrow} b$. To do so, it quantifies the unlikelihood of the smallness of the number of counterexamples $n_{a \bar{b}}(\omega)$ with respect to the independence hypothesis between the types of events $a$ and $b$. Therefore, in a search for a random model, we suppose that the types of events $a$ and $b$ are independent. Our goal is to determine the distribution of the random variable $\mathcal{N}_{a \bar{b}}$ (number of counter-examples of the rule) given the size $L$ of the sequence, the numbers $n_{a}$ and $n_{b}$ of events of types $a$ and $b$, and the size $\omega$ of the time window which is used.

We suppose that the arrival process of the events of type $b$ satisfies the following hypotheses: 
- the times between two successive occurrences of $b$ are independent random variables,

- the probability that a $b$ appears during $[t, t+\omega]$ only depends on $\omega$.

Moreover, two events of the same type cannot occur simultaneously in the sequence $S$ (see section 2.2). In these conditions, the arrival process of the events of type $b$ is a Poisson process of intensity $\lambda=\frac{n_{b}}{L}$. So, the number of $b$ appearing in a window of size $\omega$ follows Poisson's Law with parameter $\frac{\omega \cdot n_{b}}{L}$. In particular, the probability that no event of type $b$ appears during $\omega$ time units is:

$$
p=\mathrm{P}\left(\operatorname{Poisson}\left(\frac{\omega \cdot n_{b}}{L}\right)=0\right)=e^{-\frac{\omega}{L} n_{b}}
$$

Therefore, wherever it appears in the sequence, an event $a$ has the fixed probability $p$ of being a counter-example, and $1-p$ of being an example. Let us repeat $n_{a}$ times this random experiment to determine the theoretical number of counter-examples $\mathcal{N}_{a \bar{b}}$. If $\omega$ is negligible compared to $L$, then two randomly chosen windows of size $\omega$ are not likely to overlap, and we can consider that the $n_{a}$ repetitions of the experiment are independent. In these conditions, the random variable $\mathcal{N}_{a \bar{b}}$ is binomial with parameters $n_{a}$ and $p$ :

$$
\mathcal{N}_{a \bar{b}}=\operatorname{Binomial}\left(n_{a}, e^{-\frac{\omega}{L} n_{b}}\right)
$$

When permitted, this binomial distribution can be approximated by another Poisson distribution (even in the case of "weakly dependent" repetitions -see [21]) .

Definition 5 The sequential implication intensity $(\boldsymbol{S I I})$ of a rule $a \stackrel{\omega}{\longrightarrow} b$ is defined by:

$$
S I I(a \stackrel{\omega}{\longrightarrow} b)=\mathrm{P}\left(\mathcal{N}_{a \bar{b}}>n_{a \bar{b}}(\omega)\right)
$$

Numerically, we have:

$S I I(a \stackrel{\omega}{\longrightarrow} b)=1-\mathrm{P}\left(\mathcal{N}_{a \bar{b}} \leq n_{a \bar{b}}(\omega)\right)=1-\sum_{k=0}^{n_{a \bar{b}}(\omega)} \mathrm{C}_{n_{a}}^{k}\left(e^{-\frac{\omega}{L} n_{b}}\right)^{k}\left(1-e^{-\frac{\omega}{L} n_{b}}\right)^{n_{a}-k}$

\section{Properties and comparisons}

SII quantifies the unlikelihood of the smallness of the number of counterexamples $n_{a \bar{b}}(\omega)$ with respect to the independence hypothesis between the types of events $a$ and $b$. In particular, if $\operatorname{SII}(a \stackrel{\omega}{\longrightarrow} b)$ is worth 1 or 0 , then it is unlikely that the types of event $a$ and $b$ are independent (deviation from independence is significant and oriented in favor of the examples or of the counter-examples). This new index can be seen as the complement to 1 of the p-value of a hypothesis test. However, following the implication intensity, the aim here is not testing a hypothesis but actually using it as a reference to evaluate and sort the rules. 


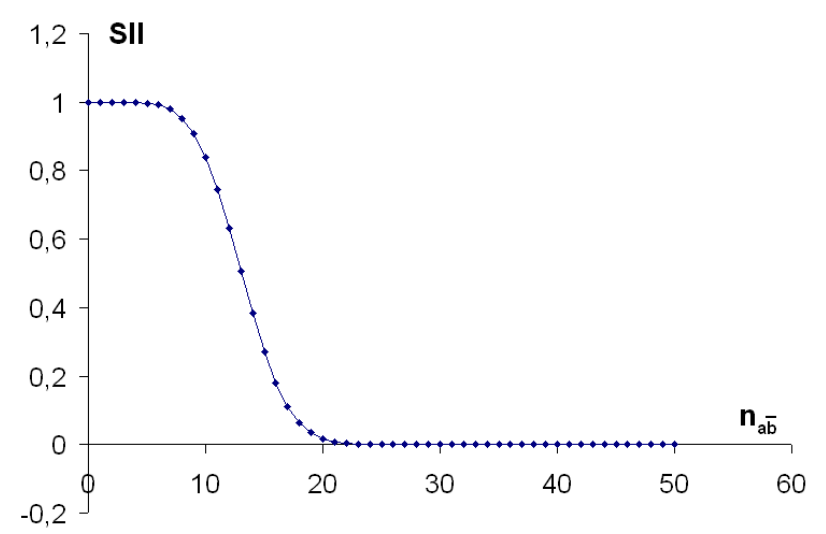

Fig. 3. $S I I$ w.r.t. the number of counter-examples.

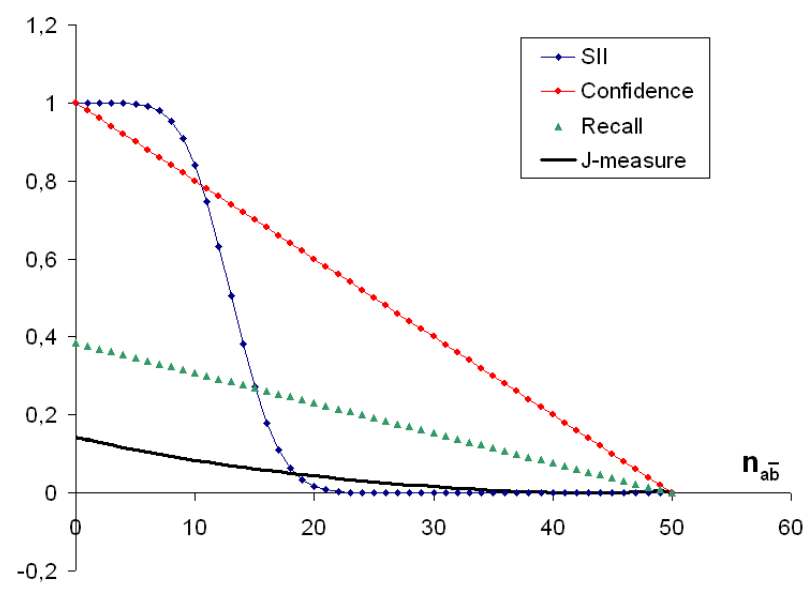

Fig. 4. $S I I$, confidence, recall, and J-mesure w.r.t. the number of counter-examples. $n_{a}=50, n_{b}=130, \omega=10, L=1000$ 
In the following, we study $S I I$ in several numerical simulations and compare it to confidence, recall, and J-measure. These simulations point out the intuitive properties of a good interestingness measure for sequential rules.

\subsection{Counter-example increase}

In this section, we study the measures when the number $n_{a \bar{b}}$ of counter-examples increases (with all other parameters constant). For a rule $a \stackrel{\omega}{\longrightarrow} b$, this can be seen as making the events $a$ and $b$ more distant in the sequence while keeping the same numbers of $a$ and $b$. This operation transforms events $a$ from examples to counter-examples.

Fig. 4 shows that SII clearly distinguishes between acceptable numbers of counter-examples (assigned to values close to 1) and non-acceptable numbers of counter-examples (assigned to values close to 0) with respect to the other parameters $n_{a}, n_{b}, \omega$, and $L$. On the contrary, confidence and recall vary linearly, while J-measure provides very little discriminative power. Due to its entropic nature, the J-measure could even increase when the number of counter-examples increases, which is disturbing for a rule interestingness measure.

\subsection{Sequence enlargement}

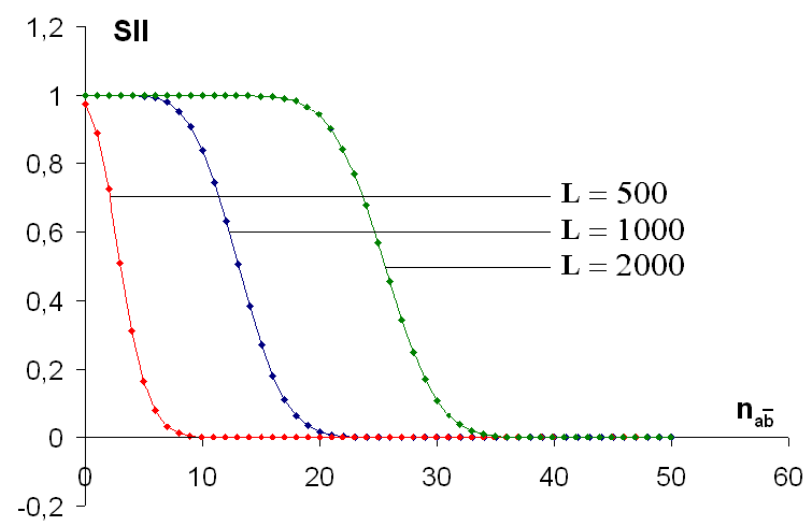

Fig. 5. $S I I$ with sequence enlargement.

$$
n_{a}=50, n_{b}=130, \omega=10
$$

We call sequence enlargement the operation which makes the sequence longer by adding new events (of new types) at the beginning or at the end. For a rule $a \stackrel{\omega}{\longrightarrow} b$, such an operation does not change the cardinalities $n_{a b}(\omega)$ and $n_{a \bar{b}}(\omega)$ since the layout of the events $a$ and $b$ remain the same. Only the size $L$ of the sequence increase. 


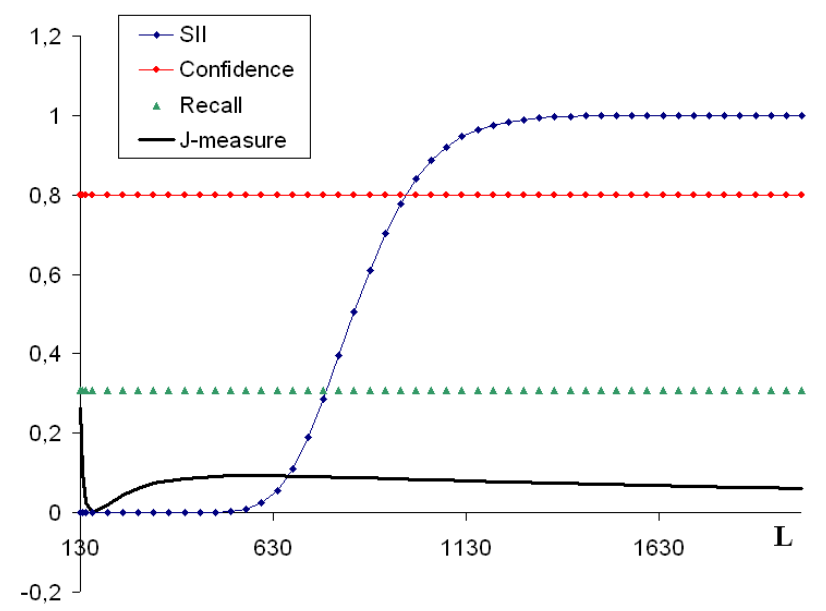

Fig. 6. $S I I$, confidence, recall, and J-mesure with sequence enlargement. $n_{a}=50, n_{b}=130, n_{a \bar{b}}=10, \omega=10$

Fig. 5 shows that $S I I$ increases with sequence enlargement. Indeed, for a given number of counter-examples, a rule is more surprising in a long sequence rather than in a short one since the $a$ and $b$ are less likely to be close in a long sequence. On the contrary, measures like confidence and recall remain unchanged since they do not take $L$ into account (see Fig. 6). The J-measure varies with $L$ but only slightly. It can even decrease with $L$, which is counter-intuitive.

\subsection{Sequence repetition}

We call sequence repetition the operation which makes the sequence longer by repeating it $\gamma$ times one after the other (we leave aside the possible side effects which could make new patterns appear by overlapping the end of a sequence and the beginning of the following repeated sequence). With this operation, the frequencies of the events $a$ and $b$ and the frequencies of the examples and counter-examples remain unchanged.

Fig. 7 shows that the values of SII are more extreme (close to 0 or 1 ) with sequence repetition. This is due to the statistical nature of the measure. Statistically, a rule is all the more significant when it is assessed on a long sequence with lots of events: the longer the sequence, the more one can trust the imbalance between examples and counter-examples observed in the sequence, and the more one can confirm the good or bad quality of the rule. On the contrary, the frequency-based measures like confidence, recall, and J-measure do not vary with sequence repetition (see Fig. 8). 


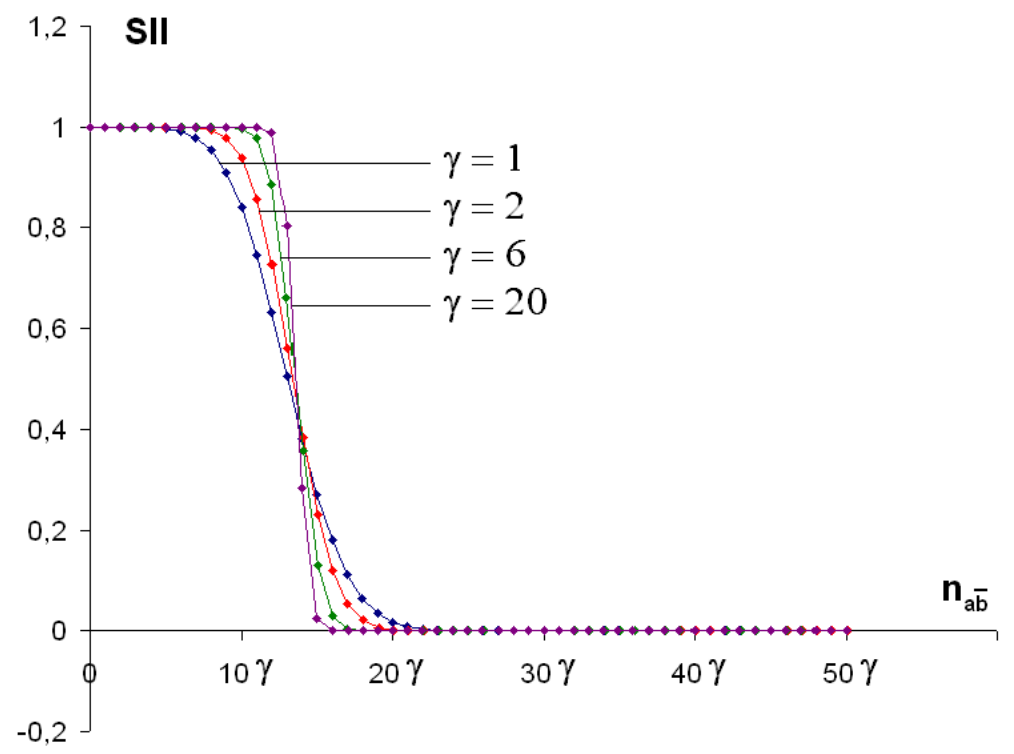

Fig. 7. $S I I$ with sequence repetition. $n_{a}=50 \times \gamma, n_{b}=130 \times \gamma, \omega=10, L=1000 \times \gamma$

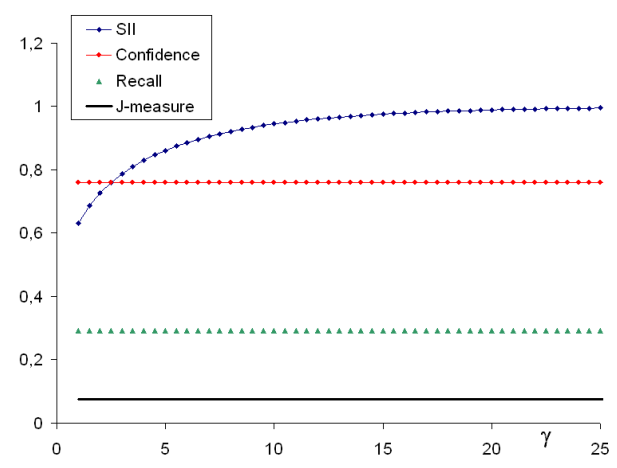

(a) $n_{a \bar{b}}=12 \times \gamma$

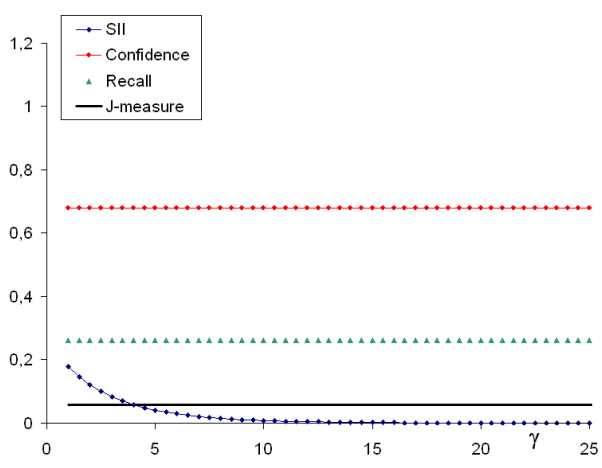

(b) $n_{a \bar{b}}=16 \times \gamma$

Fig. 8. SII, confidence, recall, and J-mesure with sequence repetition. $n_{a}=50 \times \gamma, n_{b}=130 \times \gamma, \omega=10, L=1000 \times \gamma$ 


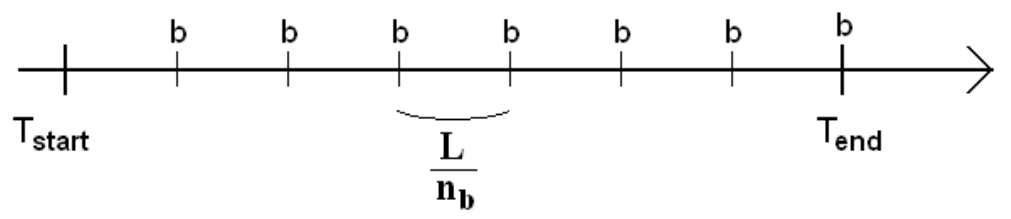

Fig. 9. A sequence where the events $b$ are regularly spread.

\subsection{Window enlargement}

Window enlargement consists of increasing the size $\omega$ of the time window. As the function $n_{a \bar{b}}(\omega)$ is unknown $\left(n_{a \bar{b}}\right.$ is given by a data mining algorithm, it depends on the data), we model it in the following way:

$$
\begin{gathered}
n_{a \bar{b}}(\omega)=n_{a}-\frac{n_{a} n_{b}}{L} \omega, \quad \text { if } \omega \leq \frac{L}{n_{b}} \\
n_{a \bar{b}}(\omega)=0, \quad \text { otherwise. }
\end{gathered}
$$

This is a simple model, considering that the number of examples observed in the sequence is proportional to $\omega: n_{a b}(\omega)=\frac{n_{a} n_{b}}{L} \omega$. The formula is based on the following postulates:

- According to definitions 2 and $3, n_{a b}$ must increase with $\omega$ and $n_{a \bar{b}}$ must decrease with $\omega$.

- If $\omega=0$ then there is no time window, and the data mining algorithm cannot find any example ${ }^{4}$. So we have $n_{a b}=0$ and $n_{a \bar{b}}=n_{a}$.

- Let us consider that the events $b$ are regularly spread over the sequence (Fig. 9 ). If $\omega \geq \frac{L}{n_{b}}$, then any event $a$ can capture at least one event $b$ within the next $\omega$ time units. So we are sure that all the events $a$ are examples, i.e. $n_{a}=n_{a b}$ and $n_{a \bar{b}}=0$.

In practice, since the events $b$ are not regularly spread over the sequence, the maximal gap between two consecutive events $b$ can be greater than $\frac{L}{n_{b}}$. So the threshold $\omega \geq \frac{L}{n_{b}}$ is not enough to be sure that $n_{a}=n_{a b}$. This is the reason why we introduce a coefficient $k$ into the function $n_{a \bar{b}}(\omega)$ :

$$
\begin{gathered}
n_{a \bar{b}}(\omega)=n_{a}-\frac{n_{a} n_{b}}{L} \frac{\omega}{k}, \quad \text { if } \omega \leq \frac{k L}{n_{b}} \\
n_{a \bar{b}}(\omega)=0, \quad \text { otherwise. }
\end{gathered}
$$

The coefficient $k$ can be seen as a non-uniformity index for the events $b$ in the sequence. We have $k=1$ only if the events $b$ are regularly spread over the sequence (Fig. 9).

\footnotetext{
${ }^{4}$ We consider that two events $a$ and $b$ occurring at the same time do not make an example.
} 


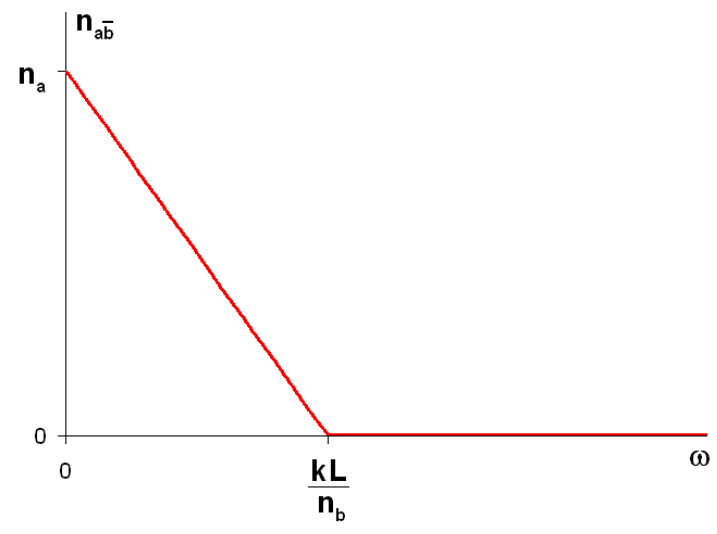

Fig. 10. Model for $n_{a \bar{b}}(\omega)$.

With this model for $n_{a \bar{b}}(\omega)$, we can now study the interestingness measures with regard to $\omega$ and $k$. Several interesting behaviors can be pointed out for $S I I$ (see illustration in Fig. 11):

- There exists a range of values for $\omega$ which allows $S I I$ to be maximized. This is intuitively satisfying ${ }^{5}$. The higher the coefficient $k$, the smaller the range of values.

- If $\omega$ is too large, then $S I I=0$. Indeed, the larger the time window, the greater the probability of observing a given series of events in the sequence, and the less significant the rule.

- As for the small values of $\omega$ (before the range of values which maximizes $S I I)$ :

- If $k \approx 1$, then $n_{a b}$ increases fast enough with $\omega$ to have $S I I$ increase (Fig. 11 at the top).

- If $k$ is larger, then $n_{a b}$ does not increase fast enough with $\omega . S I I$ decreases until $n_{a b}$ becomes more adequate (Fig. 11 at the bottom).

On the other hand, confidence (idem for recall) increases linearly with $\omega$ (see Fig. 12 with a logarithmic scale). Above all, the three measures confidence, recall, and J-measure do not tend to 0 when $\omega$ is large ${ }^{6}$. Indeed, these measures depend on $\omega$ only through $n_{a \bar{b}}$, i.e. the parameter $\omega$ does not explicitly appear in the formulas of the measures. If $\omega$ is large enough to capture all the examples, then $n_{a \bar{b}}=0$ is fixed and the three measures become constant functions (with a good value since there is no counter-example). This behavior is absolutely counter-intuitive. Only SII takes $\omega$ explicitly into account and allows rules with too large time window to be discarded.

\footnotetext{
${ }^{5}$ When using a sequence mining algorithm to discover a specific phenomenon in data, lots of time is spent to find the "right" value for the time window $\omega$.

6 This does not depend on any model chosen for $n_{a \bar{b}}(\omega)$.
} 

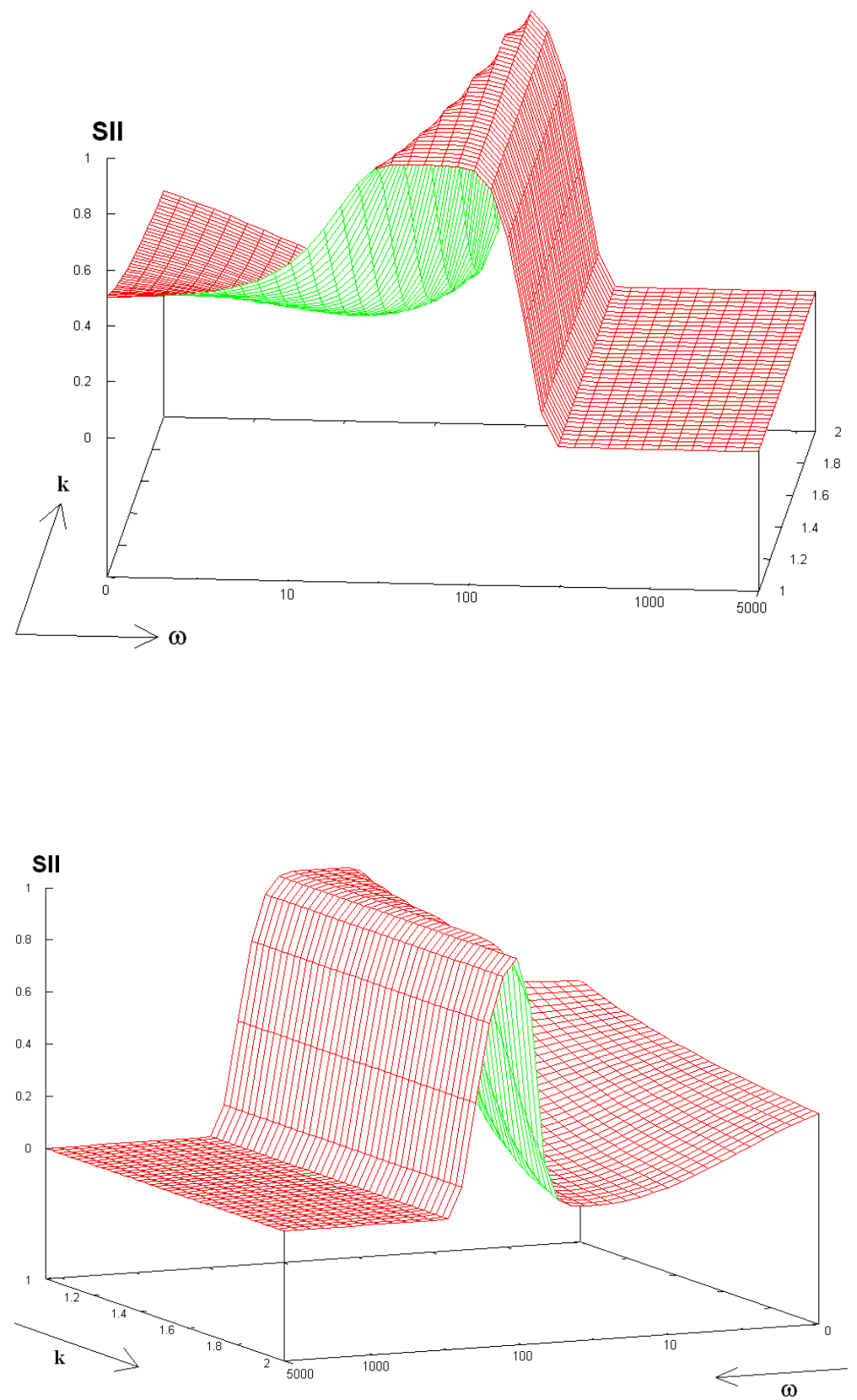

Fig. 11. $S I I$ with window enlargement. $n_{a}=50, n_{b}=100, L=5000$ 


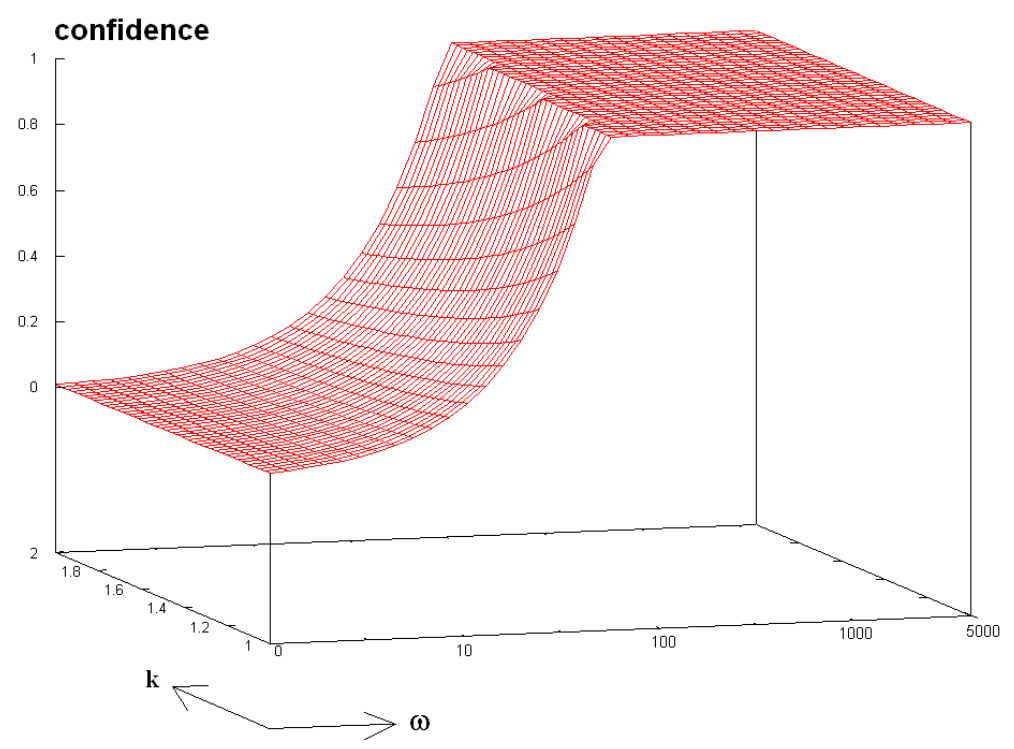

Fig. 12. Confidence with window enlargement. $n_{a}=50, n_{b}=100, L=5000$

\section{Conclusion}

In this article, we have studied the assessment of the interestingness of sequential rules. First, we have formalized the notions of sequential rule, example of a rule, and counter-example of a rule. We have then presented the Sequential Implication Intensity (SII), an original statistical measure for assessing sequential rule interestingness. $S I I$ evaluates the statistical significance of the rules in comparison with a probabilistic model. Numerical simulations show that $S I I$ has interesting features. In particular, $S I I$ is the only measure that takes sequence enlargement, sequence repetition, and window enlargement into account in an appropriate way.

To continue this research work, we are developing a rule mining platform for sequence analysis. Experimental studies of SII on real data (Yahoo Finance Stock Exchange data) will be available soon.

\section{References}

1. Mannila, H., Toivonen, H., Verkamo, A.I.: Discovering frequent episodes in sequences. In: Proceedings of the first ACM SIGKDD international conference on knowledge discovery and data mining, AAAI Press (1995) 210-215 
2. Mannila, H., Toivonen, H.: Discovering generalized episodes using minimal occurrences. In: Proceedings of the second ACM SIGKDD international conference on knowledge discovery and data mining, AAAI Press (1996) 146-151

3. Agrawal, R., Srikant, R.: Mining sequential patterns. In: Proceedings of the international conference on data engineering (ICDE), IEEE Computer Society (1995) $3-14$

4. Srikant, R., Agrawal, R.: Mining sequential patterns: generalizations and performance improvements. In: EDBT'96: Proceedings of the fifth International Conference on Extending Database Technology, Springer-Verlag (1996) 3-17

5. Mannila, H., Toivonen, H., Verkamo, A.I.: Discovery of frequent episodes in event sequences. Data Mining and Knowledge Discovery 1 (1997) 259-289

6. Das, G., Lin, K.I., Mannila, H., Renganathan, G., Smyth, P.: Rule discovery from time series. In Agrawal, R., Stolorz, P.E., Piatetsky-Shapiro, G., eds.: Proceedings of the fourth ACM SIGKDD international conference on knowledge discovery and data mining, AAAI Press (1998) 16-22

7. Höppner, F.: Learning dependencies in multivariate time series. In: Proceedings of the ECAI'02 workshop on knowledge discovery in spatio-temporal data. (2002) $25-31$

8. Sun, X., Orlowska, M.E., Zhou, X.: Finding event-oriented patterns in long temporal sequences. In Whang, K.Y., Jeon, J., Shim, K., Srivastava, J., eds.: Proceedings of the seventh Pacific-Asia conference on knowledge discovery and data mining (PAKDD2003). Volume 2637 of Lecture Notes in Computer Science., SpringerVerlag (2003) 15-26

9. Weiss, G.M.: Predicting telecommunication equipment failures from sequences of network alarms. In: Handbook of knowledge discovery and data mining. Oxford University Press, Inc. (2002) 891-896

10. Yang, J., Wang, W., Yu, P.S.: Stamp: On discovery of statistically important pattern repeats in long sequential data. In Barbará, D., Kamath, C., eds.: Proceedings of the third SIAM international conference on data mining, SIAM (2003)

11. Spiliopoulou, M.: Managing interesting rules in sequence mining. In: PKDD'99: Proceedings of the third European conference on principles of data mining and knowledge discovery, Springer-Verlag (1999) 554-560

12. Zaki, M.J.: SPADE: an efficient algorithm for mining frequent sequences. Machine Learning 42 (2001) 31-60

13. J. Han, J.P., Yan, X.: Sequential pattern mining by pattern-growth: Principles and extensions. In Chu, W.W., Lin, T.Y., eds.: Recent Advances in Data Mining and Granular Computing (Mathematical Aspects of Knowledge Discovery). SpringerVerlag (2005) 183-220

14. Joshi, M., Karypis, G., Kumar, V.: A universal formulation of sequential patterns. Technical report, University of Minnesota (1999) TR 99-021.

15. Agrawal, R., Srikant, R.: Fast algorithms for mining association rules. In Bocca, J.B., Jarke, M., Zaniolo, C., eds.: Proceedings of the twentieth international conference on very large data bases (VLDB 1994), Morgan Kaufmann (1994) 487-499

16. Hipp, J., Güntzer, U., Nakhaeizadeh, G.: Algorithms for association rule mining a general survey and comparison. SIGKDD Explorations 2 (2000) 58-64

17. Blanchard, J.: Un système de visualisation pour l'extraction, l'évaluation, et l'exploration interactives des règles d'association. $\mathrm{PhD}$ thesis, Université de Nantes (2005)

18. Blanchard, J., Guillet, F., Briand, H.: L'intensité d'implication entropique pour la recherche de règles de prédiction intéressantes dans les séquences de pannes 
d'ascenseurs. Extraction des Connaissances et Apprentissage 1 (2002) 77-88 Actes des journées Extraction et Gestion des Connaissances (EGC) 2002.

19. Gras, R.: L'implication statistique : nouvelle méthode exploratoire de données. La Pensée Sauvage Editions (1996)

20. Gras, R., Kuntz, P., Couturier, R., Guillet, F.: Une version entropique de l'intensité d'implication pour les corpus volumineux. Extraction des Connaissances et Apprentissage 1 (2001) 69-80 Actes des journées Extraction et Gestion des Connaissances (EGC) 2001.

21. Ross, S.M.: Introduction to Probability Models. (2006) 9th edition. 